\title{
Ureterolitíases obstrutivas em cães: avaliação da função renal na indicação da ureterotomia ou ureteronefrectomia
}

\author{
Obstructive ureterolithiasis in dogs: renal function evaluation in the \\ indication for ureterotomy or ureteronephrectomy
}

\section{Ureterolitiasis obstructivas en perros: evaluación de la función renal en la indicación de la ureterotomía o ureteronefrectomía}

\section{Patrícia Ferreira de Castro; ${ }^{1}$ Julia Maria Matera²}

Serviço de Cirurgia de Pequenos Animais do Hospital Veterinário da Faculdade de Medicina Veterinária e Zootecnia da Universidade de São Paulo (HOVET-FMVZ/USP). São Paulo, SP, Brasil

\section{Resumo}

Objetivo: Revisar os métodos disponíveis para avaliar a função renal, a fisiopatologia da obstrução ureteral, a nefrolitíase e a ureterolitíase, bem como os achados laboratoriais, radiográficos, ultra-sonográficos e indicações cirúrgicas relacionados à ureterolitíase obstrutiva em cães. Fontes Consultadas: Foram pesquisadas as bases de dados AGRIS de 1975 a 2004 , Biological Abstracts de 1998 a 2003, CAB Abstracts de 1990 a 2004, Medline de 1966 a 2004 e o acervo da Biblioteca da Faculdade de Medicina Veterinária e Zootecnia da Universidade de São Paulo, São Paulo, SP. Síntese dos Dados: A presença de lesões renais irreversíveis ou a funcionalidade comprometida do rim, associada à função normal do contralateral, está relacionada à decisão de realizar-se a ureteronefrectomia, em vez da ureterotomia, nas ureterolitíases obstrutivas. Em teoria, estimar a quantidade do prejuízo funcional ao rim ajuda a decidir entre preservar ou remover o rim e o ureter afetados, porém, na prática, determinar a contribuição relativa do rim acometido e do contralateral à função renal total é difícil. Conclusões: A redução da função renal no rim afetado é uma seqüela comum da obstrução ureteral, todavia, o grau de sua recuperação depois da ocorrência natural de obstrução ureteral em cães e gatos ainda não é conhecido. Na dúvida sobre a irreversibilidade da lesão renal, tentativas de desobstrução ureteral e eliminação de sua causa devem ser sempre consideradas, visto que ao menos uma recuperação parcial da função renal pode ser possível.

Palavras-chave: Ureterolitíase. Ureterotomia. Nefrectomia. Nefroureterectomia. Obstrução ureteral. Função renal. Cães.

'Médica Veterinária do Serviço de Cirurgia de Pequenos Animais do HOVET-FMVZ/USP. CRMV-SP 7944

${ }^{2}$ Professora Titular do Departamento de Cirurgia da FMVZ/USP. CRMV-SP 1050 


\section{Introdução}

Embora cálculos urinários localizados na pelve renal ou ureter sejam incomuns em cães e gatos, ${ }^{1}$ ainda assim são a causa de obstrução ureteral intraluminal mais comumente relatada em cães. ${ }^{2}$

Poucos são os casos de remoção cirúrgica de cálculos ureterais com resultados favoráveis descritos em Medicina Veterinária. ${ }^{3.4}$ Por causa do pequeno diâmetro dos ureteres e da tendência a formar constrição, a cirurgia ureteral exige destreza em sua execução. ${ }^{5}$ A litotripsia, apesar de já empregada, ${ }^{6-10}$ é pouco disponível para animais como caninos e felinos, e a ureteroscopia, utilizada para a remoção de cálculos em seres humanos, é técnica que ainda não foi adaptada para cães e gatos. ${ }^{2}$

A ureteronefrectomia é um procedimento comumente adotado nos casos de obstrução ureteral de etiologias diversas, conforme observado na literatura. ${ }^{11-13}$ A presença de lesões renais irreversíveis ou a funcionalidade comprometida do rim, associada à sua função normal contralateral, está relacionada à decisão de realizar-se a ureteronefrectomia em vez da ureterotomia nas ureterolitíases obstrutivas. ${ }^{14,15}$ Porém, determinar a contribuição relativa do rim acometido e do contralateral à função renal total é difícil. ${ }^{16}$

O objetivo deste trabalho foi o de revisar os métodos disponíveis para avaliar a função renal, a fisiopatologia da obstrução ureteral, a nefrolitíase e a ureterolitíase, os achados laboratoriais, radiográficos e ultra-sonográficos, além de fornecer indicações cirúrgicas relacionados à ureterolitíase obstrutiva em cães.

\section{Avaliação da Função Renal}

Dentre os diversos métodos usados para a avaliação da função renal - urinálise, ${ }^{17}$ prova de concentração da urina,${ }^{18}$ dosagem da uréia e creatinina séricas ${ }^{17,18}$ e testes especializados para avaliar taxa de filtração glomerular (TFG) e fluxo sanguíneo renal (FSR)-, a cintilografia renal quantitativa é aquele que permite avaliar a TFG de cada rim individualmente, entretanto, o procedimento depende, para a sua realização, de equipamentos e métodos especiais para a manipulação de radioisótopos. ${ }^{17}$ Armbrust et al. ${ }^{13}$ (1997) relataram a mensuração da TFG por meio da cintilografia nuclear em uma cadela com histórico de cálculo ureteral, diagnosticado por radiografias anteriores há pelo menos cinco meses. Este exame mostrou ser o rim ipsilateral ao cálculo ureteral responsável por menos de $5 \%$ do total da TFG, dado decisivo para a indicação da nefroureterectomia em vez da ureterotomia. Para Groshar, Issaq e Nativ (1996), a cintilografia renal fornece uma estimativa da função renal diferencial durante o episódio obstrutivo, mas não pode ser usada para predizer a função renal depois da desobstrução (apud KYLES et al., ${ }^{19}$ 1998). Lanz e Waldron ${ }^{5}$ (2000) indicam-na para a adequada avaliação do rim contralateral, antes da nefrectomia do rim comprometido.

A urinálise pode ser efetuada para: análise qualitativa da química urinária, determinação das enzimas urinárias para a avaliação da lesão renal, exame do sedimento urinário e determinação da relação entre proteína/creatinina urinária. ${ }^{17}$

A prova de concentração da urina está indicada quando repetidas amostras de urina coletadas ao acaso têm a densidade no intervalo isostenúrico; e contraindicada nos casos de uremia, debilidade e desidratação. A capacidade de concentração da urina é a primeira função a se perder após qualquer forma de obstrução do trato urinário. ${ }^{20}$

A uréia é livremente filtrada por meio dos glomérulos renais e por ser permeável em segmentos do epitélio tubular, difunde-se passivamente com a água da luz tubular, voltando para o sangue. ${ }^{17,18,21} \mathrm{~A}$ quantidade de sua absorção é inversamente proporcional ao débito do fluxo de urina pelos túbulos, ${ }^{18}$ ou seja, se o fluxo da urina é reduzido, uma quantidade maior é reabsorvida. ${ }^{18,21}$ A redução da circulação renal afeta a creatinina de modo similar à uréia, portanto, a sua mensuração fornece informação semelhante à da uréia no que diz respeito à doença renal e à obstrução e vazamento pós-renais..$^{18} \mathrm{~A}$ dosagem da creatinina plasmática é mais confiável 
do que a da uréia quando a TFG cai, porém, sua concentração não mantém relação linear com a TFG, e ainda deve-se considerar que a sua produção é determinada pela massa muscular, que varia muito entre as diferentes raças e espécies. ${ }^{22}$ Valores normais são encontrados em cães e gatos com até somente $25 \%$ da TFG. ${ }^{17}$

O prognóstico da insuficiência renal não deve ser estabelecido com base na simples determinação da uréia e da creatinina séricas, devido à influência dos fatores pré-renais, pós-renais e não-renais que podem estar relacionados. Todavia, pode fornecer informações úteis quanto à resposta da terapia e da progressão/reversibilidade das lesões renais, se realizado de forma seriada. ${ }^{23}$

A ultra-sonografia pode dar informação adicional dos detalhes das estruturas dos rins assim como a urografia intravenosa. Por fornecer análise qualitativa da função excretora renal individual, a urografia intravenosa deve ser avaliada antes da nefrectomia ou da nefrotomia. ${ }^{24}$ No entanto, deve-se considerar que sua utilidade diminui se existir azotemia $^{24}$ e que este exame não estima o número de néfrons funcionantes. ${ }^{16}$ Segundo Johnston, Walter e Feeney $^{25}$ (1995), a visualização deste exame pode estar retardada devido à pelve subaguda ou obstrução ureteral, conseqüentemente, esse atraso pode levar à interpretação de função renal pobre, indicando erroneamente situação irreversível, já que, na verdade, é reversível; quanto à ultra-sonografia, esta não facilita uma avaliação quantitativa e qualitativa da função renal, exceto pela técnica com Doppler.

O diagnóstico da disfunção renal em estágio precoce constitui-se em desafio ao profissional, pois o rim possui reserva funcional tal, que, testes-padrão laboratoriais para avaliação da função renal podem detectar anormalidades somente quando $66 \%$ do tecido funcional renal já tiver sido perdido. ${ }^{22} \mathrm{~A}$ avaliação precisa da função renal permitiria monitorização mais efetiva da sua taxa de declínio e ajudaria a determinar a eficácia da intervenção terapêutica, ${ }^{22}$ e a avaliação ideal pré-operatória de candidatos à nefrectomia incluiria avaliação da taxa de filtração glomerular renal relativa. ${ }^{16}$

\section{Fisiopatologia da Obstrução Ureteral}

A obstrução urinária é causa comum e potencialmente reversível da insuficiência renal, sendo que obstruções unilaterais agudas ou crônicas podem não ser clinicamente nítidas, permanecendo não identificadas por longos períodos, podendo resultar em lesão irreversível ao rim. Durante a obstrução unilateral, o rim não obstruído sofre alterações que podem mascarar a presença da obstrução no rim contralateral. ${ }^{20} \mathrm{~A}$ obstrução unilateral de um ureter freqüentemente resulta em hidronefrose unilateral sem evidência de prejuízo da função renal. ${ }^{26}$ Quando a obstrução aguda é completa e bilateral, se não for aliviada, a morte ocorre de $65-70$ horas $^{20}$ a três-seis dias. ${ }^{27}$

Depois de 24 horas de obstrução ureteral completa em cães, o fluxo sanguíneo renal (FSR) diminui para aproximadamente $50 \%$ do normal e retorna a aproximadamente $60 \%$ se aliviada a obstrução neste mesmo período. Já durante a obstrução unilateral crônica, diminui progressivamente, sendo, após dois meses, de aproximadamente $10 \%$. Aparentemente, o FSR retorna gradualmente ao normal no transcorrer de alguns dias após a desobstrução. Quanto à taxa de filtração glomerular (TFG), após alívio da obstrução aguda bilateral de 24 horas de duração, observa-se redução significativa de $20 \%$ do normal, permanecendo reduzida vários dias após a desobstrução. Durante a obstrução crônica, tal taxa sofre redução variável de $20 \%$ a $70 \%$, e após alívio de obstrução unilateral, dentro de seis semanas, ocorre recuperação para algo em torno de $50 \%$ a $75 \%$. Esses resultados sugerem que pode ocorrer o retorno da função renal após o alívio da obstrução crônica, e esse retorno pode ser maior se o rim mais gravemente obstruído for aliviado antes. ${ }^{20}$

A manutenção do FSR e da pressão de perfusão é necessária tanto para a filtração glomerular como para o suprimento celular de oxigênio e de nutrientes. ${ }^{28}$ Em animais com obstrução ureteral aguda, aumentam pressão e diâmetro ureterais e o peristaltismo, 
inicialmente, torna-se mais freqüente em resposta ao aumento da tensão na parede do ureter. Em seguida, com o diâmetro e a tortuosidade ureterais aumentados, o peristaltismo torna-se fraco e ineficaz para levar a urina à bexiga. $\mathrm{O}$ aumento das pressões pélvica renal e ureteral em animais com obstrução ureteral aguda ocorre como resultado da produção contínua de urina pelo rim ipsilateral. A pressão intraluminal alta é transmitida aos túbulos renais, reduzindo a diferença entre as pressões sanguínea capilar glomerular e tubular intraluminal, resultando numa TFG reduzida. O FSR também é reduzido com a vasoconstrição arterial intrarenal devido à pressão intraluminal tubular alta. Após aproximadamente 18 a 24 horas de obstrução, a pressão intraluminal diminui e pode normalizar. Entretanto, a vasoconstricção intra-renal persiste; com a obstrução crônica, a diminuição do FSR leva à perda de néfrons, atrofia do parênquima, fibrose e adicional dilatação do sistema coletor (WEISS, 1979; PFISTER; PAPANICOLAOU; YODER, 1986 apud LAMB,${ }^{29}$ 1998). Apesar de poder continuar a produção de urina pelo rim ipsilateral, a pressão intraluminal cai à medida que o ureter continua a dilatar gradualmente, num processo conhecido como ureter creep (WEISS, 1979 apud LAMB,$\left.^{29} 1998\right)$. Embora não possam ser produzidos novos néfrons e aqueles irreversivelmente lesados também não possam ser reparados, a hipertrofia funcional e morfológica dos néfrons sobreviventes freqüentemente pode compensar, de forma adequada, essa redução em seu número. Mesmo que a recuperação da função renal seja incompleta, o funcionamento adequado pode ser restabelecido. ${ }^{28}$

Estudos em cães com obstrução ureteral unilateral revelaram que a recuperação da função renal é inversamente proporcional à duração da obstrução. Em cães com obstrução renal unilateral de duas, quatro e seis semanas de duração, a recuperação da função renal foi de $38,7 \%$ após duas semanas, $9,8 \%$ após quatro semanas e $2 \%$ após seis semanas de obstrução. ${ }^{30} \mathrm{~A}$ recuperação parcial da função renal após 69 dias de completa obstrução ureteral foi observada em um caso no homem (LEWIS; PIERCE, 1962 apud KYLES et al.. ${ }^{19} 1998$ ). Para Wilson (1975; 1977 apud BJORLING; CHRISTIE, ${ }^{31} 1$ 1993), a obstrução ureteral completa por quatro semanas ou mais resulta em permanente perda da função do rim associado. Em cães de experimento submetidos à obstrução por duas semanas, o rim afetado recuperou $50 \%$ a $60 \%$ de sua função pré-obstrução, sendo considerado quatro semanas o tempo máximo de obstrução completa com alguma probabilidade de retorno à função renal adequada. ${ }^{3}$

Estudos experimentais em cães revelaram que, se aliviada a obstrução ureteral dentro de dez semanas após sua ocorrência, o segmento dilatado retorna ao seu diâmetro e à sua forma normais. ${ }^{31} \mathrm{Em}$ uma cadela submetida à anastomose ureteral unilateral devido à ruptura traumática, severos hidronefrose e hidroureter observados ao urograma e à ultra-sonografia 13 dias posteriores à cirurgia apresentaram melhora após dez semanas. Aos 11 meses após o trauma original, as imagens do rim e ureter acometidos eram muito próximas da normalidade. ${ }^{32}$

Dois casos de ureterotomia para remoção de ureterólitos com resultados satisfatórios em dois cães, com retorno do rim ao tamanho normal ou subnormal também foram descritos. ${ }^{3}$ Em outro trabalho, dois casos de ureterotomia em cães com acompanhamento clínico, laboratorial, radiográfico e ultra-sonográfico até 210 dias após intervenção cirúrgica para remoção dos ureterólitos (Figuras 1 e 2) demonstraram ausência de sinais de estenose ou vazamento nos sítios operados e diminuição da dimensão renal e do limite córtico-medular, sugerindo o comprometimento da funcionalidade do rim acometido. ${ }^{4}$

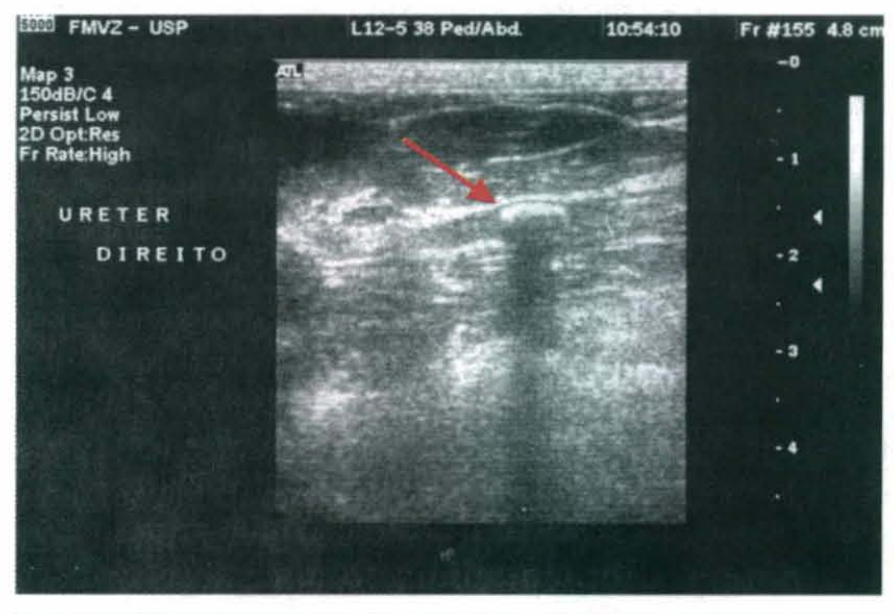

Figura 1 - Imagem ultra-sonográfica sugestiva de urolitíase (seta) localizada em ureter direito de fêmea canina da raça Schnauzer, com sete anos de idade 


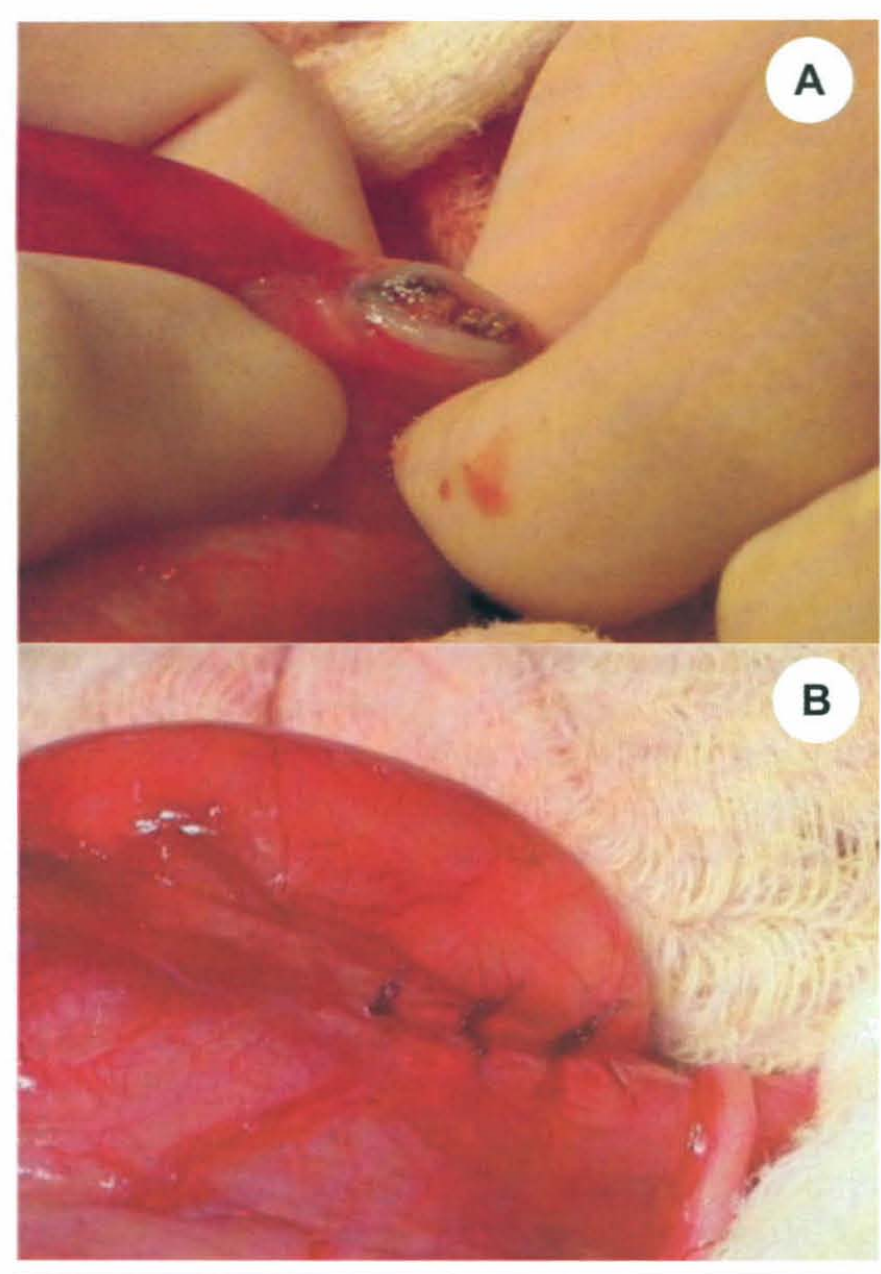

Figura 2 - Imagem fotográfica de ureterolitíase em cão macho SRD: A - Ureterotomia direita para a retirada de urólitos em inserção uretero-vesical; B - Síntese ureteral no plano longitudinal com fio monofilamento de poligliconato no padrão pontos simples interrompidos

$\operatorname{McEvoy}^{33}$ (1994) relatou a recuperação de uma cadela com obstrução ureteral bilateral severa pósovariohisterectomia, com satisfatório retorno da função renal em um rim que permaneceu por, no mínimo, quatro dias com obstrução do seu fluxo. Um ureter foi reimplantado na bexiga e o outro, por não apresentar viabilidade, resultou em ureteronefrectomia. $\mathrm{O}$ autor referiu-se à melhora da poliúria pós-operatória que persistiu por cinco semanas, sem mencionar, contudo, a evolução tardia do procedimento cirúrgico. Já em outro relato, dois cães com obstrução ureteral unilateral por pólipos fibroepiteliais foram submetidos à ureteronefrectomia, apesar de Burton et al. ${ }^{12}$ citarem que, no homem, a excisão local dos pólipos via ureterotomia, ureteroscopia e cirurgia a laser tem permitido a preservação do rim. Adicionalmente é necessário considerar também que a dilatação ureteral e a hidronefrose apresentam melhora no pósoperatório e que, em $37 \%$ dos pacientes humanos com esta afecção realizou-se desnecessariamente a nefrectomia. Afirmam que, nestes dois casos, o diagnóstico histopatológico pré-operatório poderia ter sido realizado sem, contudo, alterar a conduta, devido ao avançado estágio da hidronefrose.

\section{Litíase Renal e Ureteral}

A nefrolitíase ${ }^{1}$ e a ureterolitíase $e^{1,31}$ são condições raras nos cães e gatos. Aproximadamente 97\% dos pacientes humanos com cálculos apresentam nefrolitíase, enquanto menos de $4 \%$ dos casos de urolitíases em cães e gatos são de cálculos renais, desconhecendo-se a razão para tal disparidade. ${ }^{1}$ De um total de 17.610 urólitos caninos analisados por métodos quantitativos no Minnesota Urolith Center, apenas $226(1,3 \%)$ eram nefrólitos. ${ }^{34}$ Em estudo realizado por Ling et al. ${ }^{1}$ (1998), os cálculos de origem renal representaram $2,88 \%$ do total de cálculos urinários submetidos à análise nos cães, e 4,95\% do total nos gatos.

É amplamente aceito que a urolitíase é uma doença multifatorial, ${ }^{35}$ constituindo-se como fatores que contribuem para a formação de urólitos a alta concentração de cristalóides na urina, a diminuição na concentração de inibidores de cristalização urinária, ${ }^{15,26,35,36} \mathrm{o} \mathrm{pH}$ urinário favorável, ${ }^{15,26,35} \mathrm{a}$ infecção, ${ }^{15,26,36}$ a presença de um núcleo sobre o qual a cristalização possa ocorrer ${ }^{26}$ e certas anormalidades metabólicas; ${ }^{36}$ porém, sem a supersaturação, nenhum dos outros fatores pode operar. ${ }^{35}$

$\mathrm{O}$ valor do $\mathrm{pH}$ urinário sugere a provável composição mineral do cálculo em cães e gatos, ${ }^{34} \mathrm{e}$ a análise dos cálculos removidos e a urocultura visam a um tratamento médico direcionado para prevenir recidivas. ${ }^{15}$ Em geral, é difícil eliminar infecções do trato urinário se os urólitos estiverem presentes ${ }^{15} \mathrm{e}$, assim, doenças persistentes do trato urinário e urolitíases recorrentes podem ocorrer se a remoção cirúrgica constituir-se na única forma de tratamento. ${ }^{14}$ 
Estudo retrospectivo mostrou que, de um total de 438 cães tratados cirurgicamente para urolitíase, 111 pacientes apresentaram 155 recorrências. ${ }^{34}$ Desse modo, a probabilidade de recorrência de urólitos após a terapia médica ou cirúrgica tem tido prognóstico reservado, dada a influência de muitas variáveis, tais quais: método diagnóstico, remoção do urólito inadequada, infecção, manejo pelo proprietário. ${ }^{34}$

\section{Ureterolitíase Obstrutiva: achados laboratoriais, radiográficos e ultra- sonográficos}

Achados anormais na urinálise de animais com obstrução ureteral são inespecíficos, no entanto, é comum a hematúria microscópica. Cristais em amostra de urina fresca podem dar idéia do tipo de ureterólito, se presente, porém, tais cristais também podem ocorrer em animais normais. Durante a obstrução crônica, devido ao déficit de concentração, a produção de urina diluída pelo rim afetado poderá ocorrer. Todavia, a urina colhida da bexiga estará concentrada se o outro rim estiver mantendo sua capacidade de concentração. Igualmente, as concentrações séricas de uréia e creatinina podem estar dentro dos limites normais. ${ }^{37}$

A radiografia de todo o trato urinário deve ser sempre realizada para determinar a localização e o número de urólitos. ${ }^{14,34} \mathrm{~A}$ maioria dos cálculos renal e ureteral é radiopaca, ${ }^{15}$ no entanto, radiografias contrastadas também podem ser necessárias para o diagnóstico. ${ }^{14}$ Hidroureter ou hidronefrose devem ser avaliados preferivelmente pela ultra-sonografia ${ }^{37} \mathrm{ou}$ pela urografia excretora ${ }^{15}$ e constituem-se em alterações altamente sugestivas de imobilidade de ureterólitos. ${ }^{14}$

\section{Ureterolitíases: ureterotomia ou ureteronefrectomia}

Caywood e Osborne ${ }^{14}$ (1986) indicam a ureterotomia nos pacientes que não apresentem dano irreversível ao ureter e rim. Osborne et al. ${ }^{34}$ (1995) referem que a cirurgia deve ser considerada no caso de urólitos em lúmen ureteral, especialmente se associados à infecção e, dentre os procedimentos mais comumente utilizados em cães - ureterolitotomia, ureterectomia e nefrectomia -, este último está indicado nos casos de severa hidronefrose unilateral, pionefrose, múltiplos abscessos renais, nefrolitíase unilateral obstrutiva recorrente, desde que o rim contralateral seja suficientemente funcional e, portanto, capaz de manter a homeostasia.

Dupre et al. ${ }^{3}$ (1990) citam que afecções como cálculos ureterais que não se movimentam em 24 horas, hidroureter ou hidronefrose, têm indicação de ureterotomia. Afirmam que a remoção precoce dos cálculos, com técnica cirúrgica adequada, pode restabelecer as funções renal e ureteral, evitando-se assim a nefroureterectomia; e a ureterotomia deve ser tentada se o rim afetado não apresenta prejuízo irreversível.

Kyles, Aronsohn e Stone ${ }^{38}$ (1996), ao comentarem sobre as possíveis complicações póscirúrgicas da ureteronefrectomia, citam a falência renal do rim remanescente. Relacionam a falência renal a disfunções pré-existentes e/ou a isquemia secundária à anestesia, e dizem que uma redução superior a $50 \%$ na taxa de filtração glomerular resultará em azotemia renal. Kyles et al. ${ }^{19}$ (1998) mencionam que um prognóstico preciso do potencial da função renal do rim comprometido poderia ajudar na tomada da decisão pela nefroureterectomia ou pela remoção dos ureterólitos, e que o grau de dilatação da pelve não está relacionado com a quantidade do prejuízo funcional.

Segundo Fossum ${ }^{15}$ (1997), a indicação da remoção de todos os cálculos renais e ureterais é controversa. A remoção cirúrgica de cálculos renais e ureterais deveria ser considerada quando eles estão infectados ou causando obstrução. A cirurgia deveria ser realizada tão logo quanto possível, uma vez que o paciente esteja estabilizado, para prevenir dano renal irreversível.

Para Stone e $\mathrm{Kyles}^{37}$ (2000), a remoção cirúrgica de ureterólitos é indicada quando a obstrução, 
parcial ou completa, do ureter ocorre, evidenciada pela hidronefrose e hidroureter proximal ao cálculo ou pela imobilidade do ureterólito. Tal obstrução pode ser determinada por repetidos exames radiográficos e/ou ultra-sonográficos. Fatores que também influenciam na decisão de remover ureterólitos são a presença de infecção, outros cálculos no trato urinário e o grau de função renal no rim afetado.

A nefrectomia constitui-se em tratamento irreversível para doença renal unilateral quando for descartada qualquer contribuição para a função renal por parte daquele rim (STONE; GOOKIN,${ }^{16} 2000$; KYLES et al., ${ }^{19}$ 1998). Durante a afecção aguda, a presença de um rim não-funcional pode fornecer estímulo maior para a hipertrofia compensatória no rim oposto, do que aquela gerada se o rim não-funcional fosse removido (STONE; GOOKIN, ${ }^{16} 2000$ ).

\section{Considerações Finais}

Em teoria, estimar a quantidade do prejuízo funcional ao rim ajuda a decidir entre preservar ou remover o rim e o ureter afetados, porém, na prática, determinar a contribuição relativa do rim acometido e do contralateral à função renal total é difícil, assim como determinar o seu potencial de recuperação, pois o grau de dilatação não está correlacionado ao grau de prejuízo funcional, e os exames disponíveis para avaliar a função renal não permitem assegurar, num breve espaço de tempo pré-operatório, o quanto tal órgão ainda é funcional.

O potencial para recuperação da função renal depende da duração e extensão da obstrução, bem como da presença de infecção do trato urinário. A redução da função renal no rim afetado é seqüela comum da obstrução ureteral, contudo, o grau de sua recuperação depois da ocorrência natural de obstrução ureteral em cães e gatos ainda não é conhecido.

Na dúvida sobre a irreversibilidade da lesão renal, tentativas de desobstrução ureteral e eliminação de sua causa deveriam ser sempre consideradas, visto que, ao menos, uma recuperação parcial da função renal é possível. Sugere-se, assim, a ureterotomia, em tais casos.

\section{Abstract}

Objective: To review the methods of renal function evaluation available, the pathophysiology of ureteral obstruction, the nephrolithiasis and ureterolithiasis, the laboratory, radiographic and ultrasonographic findings and the surgical indications related to canine obstructive ureterolithiasis. Data Sources: AGRIS (from 1975 to 200), Biological Abstracts (from 1998 to 2003), CAB Abstracts (from 1990 to 2004) and Medline (from 1966 to 2004) databases and the collection of the library of the Faculdade de Medicina Veterinária e Zootecnia da Universidade de São Paulo (School of Veterinary Medicine and Animal Science of the University of São Paulo), São Paulo, Brazil, were consulted. Data Synthesis: Irreversible damage or compromised kidney function, associated to a normal functioning contralateral kidney, supports the decision for performing an ureteronephrectomy instead of an ureterotomy when obstructive ureterolithiasis occurs. In theory, estimating the amount of functional damage to the kidney helps to decide whether to preserve or remove the affected kidney and ureter; however, estimating the relative contribution of the affected and the contralateral kidney to the total renal function is difficult in practice. Conclusions: The reduced function of the affected kidney is a common consequence of ureteral obstruction, but the degree of recovery after the natural occurrence of this disease in dogs and cats has not yet been established. When in doubt as to the irreversibility of the renal damage, attempts to achieve ureteral desobstruction and treatment of the underlying causes should always be considered, once at least a partial recovery of the renal function may be possible.

Keywords: Ureterolithiasis. Ureterotomy. Nephrectomy. Nephroureterectomy. Ureteral obstruction. Renal function. Dogs. 


\section{Resumen}

Objetivo: Revisar los métodos disponibles para evaluar la función renal, la fisiopatología de la obstrucción del uréter, la nefrolitiasis y la ureterolitiasis, los datos laboratoriales, radiográficos y ecográficos e indicaciones quirúrgicas relacionados a la ureterolitiasis obstructiva en perros. Fuentes Consultadas: Se investigaron las bases de datos AGRIS de 1975 a 2004, Biological Abstracts de 1998 a 2003, CAB Abstracts de 1990 a 2004, Medline de 1966 a 2004 y el acervo de la Biblioteca de la Faculdad de Medicina Veterinaria y Zootecnia de la Universidad de São Paulo, São Paulo, SP, Brasil. Síntesis de los Datos: Los daños irreversibles o el comprometimiento de la función del riñón, asociado a la función normal del contralateral, están relacionados a la decisión de realizar la ureteronefrectomía en vez de ureterotomía, en los casos de ureterolitiasis obstructivas. En teoría, calcular la cantidad de daño funcional del riñón, ayuda a decidir entre preservar o retirar el riñón y uréter afectados, pero en la práctica, determinar la contribución relativa del riñón comprometido y del contralateral a la función renal total se hace dificil. Conclusiones: La reducción de la función renal en el riñón afectado es una secuela común de la obstrucción natural del uréter, pero el grado de recuperación después de la obstrucción del uréter en perros y gatos se desconoce. En la duda sobre la irreversibilidad de la lesión renal, intentos de desobstrucción del uréter y eliminación de su causa deben ser siempre consideradas, porque al menos una recuperación parcial de la función renal es posible.

Palabras-clave: Ureterolitiasis. Ureterotomía. Nefrectomía. Nefroureterectomía. Obstrucción ureteral. Función renal. Perros.

\section{Referências}

1. LING, G. V. et al. Renal calculi in dogs and cats: prevalence, mineral type, breed, age, and gender interrelationships (1981-1993). Journal of Veterinary Internal Medicine, v. 12, p. 11-21, 1998.

2. HARDIE, E. M.; KYLES, A. E. Management of ureteral obstruction. Veterinary Clinics of North America: Small Animal Practice, v. 34, p. 889-1010. 2004.

3. DUPRE, G. P.; DEE, L. G.; DEE, J. F. Ureterotomies for treatment of ureterolithiasis in two dogs. Journal of the American Animal Hospital Association, v. 26, p. 500-504, 1990.

4. CASTRO, P. F. et al. Ureterotomia como tratamento de obstrução ureteral em dois cães. Brazilian Journal of Veterinary Research and Animal Science, v. 40, p. 158, 2003. Suplemento.

5. LANZ, O. I.; WALDRON, D. R. Renal and ureteral surgery in dogs. Clinical Techniques in Small Animal Practice, v. 15, p.1-10, 2000.

6. BAILEY, G; BURK, R. L. Dry extracorporeal shock wave lithotripsy for treatment of ureterolithiasis and nephrolithiasis in a dog. Journal of the American Veterinary Medical Association, v. 207, p. 592-595, 1995.
7. BLOCK, G. et al. Use of extracorporeal shock wave lithotripsy for treatment of nephrolithiasis and ureterolithiasis in five dogs. Journal of the American Veterinary Medical Association, v. 208, p. 531-536, 1996.

8. LOSKE, A. M.; PRIETO, F. E.; LÓPEZ, J. A. Primer tratamiento de litotripsia extracorporal en un perro usando un generador de ondas de choque experimental hecho en México. Veterinaria Mexico, v. 27, p.4148, 1996.

9. ADAMS, L. G.; SENIOR, D. F. Electrohydraulic and extracorporeal shock-wave lithotripsy. Veterinary Clinics of North America: Small Animal Practice, v. 29, p. 293-302, 1999.

10. LANE, I. F. Lithotripsy: an update on urologic applications in small animals. Veterinary Clinics of North America: Small Animal Practice, v. 34, p. 1011-1025. 2004.

11. CRAWFORD, M. A.; TURK, M. A. M. Ureteral obstruction associated with proliferative ureteritis in a dog. Journal of the American Veterinary Medical Association, v. 184, p. 586-588, 1984. 
12. BURTON, C. A. et al. Ureteric fibroepithelial polyps in two dogs. Journal of Small Animal Practice, v. 35 , p. $593-596,1994$.

13. ARMBRUST, L. et al. Radiographic diagnosis: canine ureteral calculus. Veterinary Radiology Ultrasound, v. 38, p. 360-362, 1997.

14. CAYWOOD, D. D.; OSBORNE, C. A. Surgical removal of canine uroliths. Veterinary Clinics of North America: Small Animal Practice, v. 16, p. 389-399, 1986.

15. FOSSUM, T. W. Surgery of the kidney and ureter. In:__. Small animal surgery. Saint Louis: Mosby, 1997. p. 461-480.

16. STONE, E. A.; GOOKIN, J. Urinary disorders: indications for nephrectomy and nephrotomy. In: BONAGURA, J. D. Kirk's current veterinary therapy. Small animal practice. Philadelphia: Saunders, 2000. v. 13, p. 866-868.

17. BROWN, S. A. Physiology of the urinary tract. In: SLATTER, D. Textbook of small animal surgery. 2. ed. Philadelphia: Saunders, 1993. p.1384-1395.

18. DUNCAN, J. R.; PRASSE, K. W. Aparelho urinário. In: Patologia clínica veterinária. Rio de Janeiro: Guanabara Koogan, 1982. p. 89-107.

19. KYLES, A. E. et al. Diagnosis and surgical management of obstructive ureteral calculi in cats: 11 cases (1993-1996). Journal of the American Veterinary Medical Association, v. 213, p. 1150 1156, 1998.

20. BOVÉE, K. C.; ROSIN, A. E.; HART, B. L. Pathophysiology and therapeutics of urinary tract disorders. In: SLATTER, D. Textbook of small animal surgery. 2. ed. Philadelphia: Saunders, 1993. p.1396-1415.

21. GRAUER, F. G. Manifestações clínicas dos distúrbios urinários. In: NELSON, R. W.; COUTO, C. G. Fundamentos de medicina interna de pequenos animais. Rio de Janeiro: Guanabara Koogan, 1994. p. 331-343.
22. ELLIOTT, J. Assessment of renal function: what can be done in practice. In: WSAVA CONGRESS, 27. 2002, Granada. Proceedings... Disponível em: h t p p : / / w w w. vin.com/proceeding / Proceedings.plx?CID=WSAVA2002\&PID $=2704$. Acesso: 21 mar. 2003.

23. POLZIN, D. J.; OSBORNE, C. A. Prognosis of renal disease, renal failure, and uremia. In: OSBORNE, C. A.; FINCO, D. R. Canine and feline nephrology and urology. Philadelphia: Williams \& Wilkins, 1995. p. 505-507.

24. GRAUER, F. G. Testes diagnósticos para o sistema urinário. In: NELSON, R. W.; COUTO, C. G. Fundamentos de medicina interna de pequenos animais. Rio de Janeiro: Guanabara Koogan, 1994. p. 344-349.

25. JOHNSTON, G. R.; WALTER, P. A.; FEENEY, D. A. Diagnostic imaging of the urinary tract. In: OSBORNE, C. A.; FINCO, D. R. Canine and feline nephrology and urology. Philadelphia: Williams \& Wilkins, 1995. p. 230-276.

26. GRAUER, F. G. Urolitíase em caninos. In: NELSON, R. W.; COUTO, C. G. Fundamentos de medicina interna de pequenos animais. Rio de Janeiro: Guanabara Koogan, 1994. p. 369-374.

27. OSBORNE, C. A et al. A clinician's analysis of urinalysis. In: OSBORNE, C. A.; FINCO, D. R. Canine and feline nephrology and urology. Philadelphia: Williams \& Wilkins, 1995. p. 136-205.

28. GRAUER, F. G. Insuficiência renal. In: NELSON, R. W.; COUTO, C. G. Fundamentos de medicina interna de pequenos animais. Rio de Janeiro: Guanabara Koogan, 1994. p. 355-363.

29. LAMB, C. R. Ultrasonography of the ureters. Veterinary Clinics of North America: Small Animal Practice, v. 28, p. 823-848, 1998.

30. OSBORNE, C. A.; POLZIN, D. J. Nonsurgical management of canine obstructive urolithopathy. Veterinary Clinics of North America: Small Animal Practice, v. 16, p. 333-347, 1986. 
31. BJORLING, D. E.; CHRISTIE, B. A. Ureters. In: SLATTER, D. Textbook of small animal surgery. 2. ed. Philadelphia: Saunders, 1993. p. 1443-1450.

32. CHAMBERS, J. N.; SELCER, B. A.; BARSANTI, J. A. Recovery from severe hydroureter and hydronephrosis after ureteral anatomosis in a dog. Journal of the American Veterinary Medical Association, v. 191, p. 1589-1592, 1987.

33. McEVOY, F. J. Iatrogenic renal obstruction in a dog. Veterinary Record, v. 135, p. 457-458, 1994.

34. OSBORNE, C. A. et al. Canine and feline urolithiases: relationship of etiopathogenesis to treatment and prevention. In: OSBORNE, C. A.; FINCO, D. R. Canine and feline nephrology and urology. Philadelphia: Williams \& Wilkins, 1995. p. $798-888$.
35. KHAN, S. R. Calcium oxalate urolithiasis in rat. In: JONES, T. C.; HARD, G. C.; MOHR, U. Urinary system. 2. ed. Berlin: Springer, 1998. p. 431-438.

36. MEYER, J. L. Physiocochemistry of stone formation. In: RESNICK, M. I.; PAK C. Y. C. Urolithiasis: a medical and surgical reference. Philadelphia: Saunders, 1990. p. 11-34.

37. STONE, E. A.; KYLES, A. E. Urinary disorders: diagnosis and management of ureteral obstruction. In: BONAGURA, J. D. Kirk's current veterinary therapy. Small animal practice. Philadelphia: Saunders Company, 2000. v. 13, p. 868-871.

38. KYlES, A. E.; ARONSOHN, M.; STONE, E. A. Urogenital surgery. In: LIPOWITZ, A. J. et al. Complications in small animal surgery: diagnosis, management, prevention. Baltimore: Williams \& Wilkins, 1996. p. 455-475. 